\title{
Poliposis de colon
}

\author{
Rodolfo Avendaño H , Patricio Fernández A², María Fernanda Deichler V²
}

\begin{abstract}
RESUMEN
El cáncer colo-rectal (CCR) es la segunda causa de muerte por cáncer en los países occidentales. En el año 2004 se diagnosticaron aproximadamente 160.000 casos nuevos en EEUU, reportándose alrededor de 60.000 muertes por esta causa. En nuestro país se ha reportado un aumento dramático en la incidencia de este tipo de tumores, sin embargo, una de las principales características de éstos, es su buen pronóstico, logrando cifras de sobre vida a 5 años de hasta un 80\%. Para alcanzar estos resultados es fundamental el diagnóstico precoz. En este sentido es muy importante tener una actitud agresiva frente a la pesquisa e identificación de pacientes con riesgo de desarrollar un CCR. Se ha considerado que los pólipos de colon son un factor de riesgo importante para desarrollar un CCR. De ahi deriva la importancia de su conocimiento, detección y extracción. El presente artículo tiene como objetivo presentar una actualización de los distintos tipos de pólipos excluyendo a la poliposis adenomatosa familiar, entidad que creemos merecería un artículo propio y por lo tanto escapa de los objetivos de la presente revisión. (Palabras claves/Key words: Poliposis colónica/colon polyposis; Polipos de colon/colonic polyps; cancer colorectal/colorectal cancer).
\end{abstract}

\section{INTRODUCCIÓN}

El cáncer colo-rectal (CCR) es la segunda causa de muerte por cáncer en los países occidentales' ${ }^{1}$. En el año 2004 se diagnosticaron aproximadamente 160.000 casos nuevos en EEUU², reportándose alrededor de 60.000 muertes por esta causa.

En nuestro país, si bien no existe un sistema de registro nacional de tumores, se ha reportado recientemente un aumento dramático en la incidencia de este tipo de tumores, con un incremento en el fallecimiento por CCR que ascendió de 486 personas en 1990 a 976 personas en el $2003^{3}$.

Una de las principales características de estos tumores es su buen pronóstico, logrando cifras de sobre vida a 5 años de hasta un $80 \%$. Para alcanzar esta sobrevida es fundamental el diagnóstico precoz. En este sentido es muy importante tener una actitud agresiva frente a la pesquisa e identificación de pacientes con factores de riesgo de desarrollar un CCR.

Desde que Dukes en $1926^{4}$, y luego Jackman y Mayo en $1951^{5}$ establecieran la progresión de adenoma a carcinoma, se ha considerado que los pólipos de colon son un factor de riesgo importante para desarrollar un CCR. De ahí deriva la importancia de su conocimiento, detección y extracción.

\section{Definición:}

El término pólipo deriva del griego poli (muchos) y pous (pies). Se aplica para cualquier tumor o tumefacción que surge de la mucosa del intestino, ya sea pedunculado o sésil.

Estrictamente hablando éste término no da indicación alguna sobre su estructura, comportamiento ni riesgo de malignización. Debido a lo anterior de los ha clasificado según sus características.

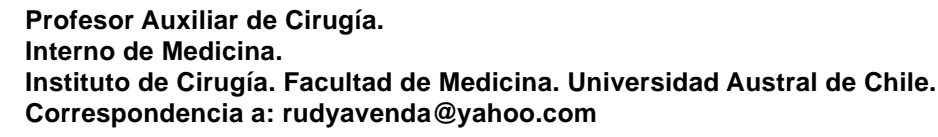




\section{Clasificación:}

1) Neoplásicos: Adenoma tubular, túbulo velloso y velloso.

2) Hamartomatosos: Pólipo juveniles, PeutzJeghers, Cronkhite-canada, Enfermedad de Cowden.

3) Inflamatorios: Pólipo inflamatorio o pseudos pólipo, pólipo benigno linfoídeo.

4) Hiperplasicos.

Los pólipos son frecuentes en la población general. Estudios realizados en cadáveres muestran una frecuencia de entre 30 a $50 \%$ en la población general, el $90 \%$ es menor a $1 \mathrm{~cm}$, el $66 \%$ corresponden a adenoma, el $11 \%$ a pólipos hiperplásicos y el $24 \%$ al resto. Sólo el 1 al $4 \%$ correspondieron a cáncer.

\section{1) Pólipos neoplásicos:}

Son crecimientos epiteliales compuestos por glándulas anormales de la mucosa del colon. Se clasifican dependiendo de su componente velloso:

a) Adenomas tubulares: $0-25 \%$ de componente velloso. Corresponden al $75 \%$ de los pólipos. Sólo el $5 \%$ son malignos al momento del diagnóstico.

b) Adenoma túbulo velloso: 25 a $75 \%$ de componente velloso. Corresponden al $15 \%$. El $20 \%$ son malignos al momento de diagnóstico.

c) Adenoma velloso: 75 a $100 \%$ de componente velloso. Corresponden al $10 \%$ de los pólipos. Entre el 35 y el $40 \%$ son malignos.

Además del tipo histológico, el tamaño y el grado de diplasia se asocian con el potencial de malignización. Así, por ejemplo, el 1\% de los menores de $1 \mathrm{~cm}$ son malignos, el $10 \%$ de los entre 1 y $2 \mathrm{~cm}$ y el $50 \%$ de los sobre $2 \mathrm{~cm}^{6}$

El $60 \%$ de los adenomas se encuentran distales al ángulo esplénico.

El $60 \%$ son lesiones únicas, y el $40 \%$ restante presentan pólipos múltiples.

Esta demostrado que la remoción sistemática del los pólipos de colon reduce en forma importante la incidencia de CCR. El 24\% de los pacientes a los que se les deja pólipos in situ desarrollaran un CCR dentro de 20 años. ${ }^{7}$

\section{Secuencia Adenoma -Carcinoma:}

La aparición de CCR desarrollado a partir de un adenoma benigno fue establecida por primera vez por Dukes en el Hospital St Marks en 1926, y ratificado por Mayo en 1951. Este concepto esta hoy en día totalmente aceptado y es la base de la prevención secundaria a través de polipectomías colonoscópicas.

\section{Genética Molecular}

Un adenoma se produce por proliferación del epitelio que comienza en una célula. Esto se produce por una serie de mutaciones secuenciales que pueden terminar incluso en la formación de un CCR.

La primera mutación de la secuencia se produce en el gen APC (adenomatous polyposis coli) ubicado en el cromosoma 5q. Debido a la mutación, éste gen es inactivado permitiendo la proliferación incontrolada de la célula. Debido al aumento en la velocidad de proliferación se produce un aumento en la posibilidad de aparición de nuevas mutaciones.

El siguiente paso es la hipometilación del DNA. Esto favorece aún más la proliferación celular, permitiendo la formación del adenoma. Esta hipometilación favorece la activación de un proto-oncogen del cromosoma 12 (K-ras) permitiendo la aparición de la displasia. La progresión a displasia de alto grado se produce por la mutación del gen DCC (deleted in colon cancer), ubicado en el gen 18. Finalmente el cáncer se produce al aparecer una mutación en el cromosoma 17p, específicamente en el gen $p 53$. (Figura 1).

\section{Diagnóstico}

Si bien el síntoma más frecuentemente asociado es la rectorragia, en la gran mayoría de los casos son asintomáticos, y son diagnosticados en forma incidental durante la realización de un enema baritado o de una colonoscopía.

Otros síntomas asociados son la diarrea (en el caso de un adenoma velloso), dolor abdominal intermitente (poco frecuente) y anemia leve a moderada.

Los pólipos pueden desarrollarse en dos formas: sésiles o pedunculados.

En 1985 Muto describió un tercer tipo de adenomas que llamó "adenomas planos", que se caracterizan por ser pequeños y planos, y por lo tanto difíciles de diagnosticar. Sin embargo tienen un potencial de malignización mayor que el resto de los pólipos adenomatosos, ya que se asocian a cáncer en el $6 \%$ de los adenomas planos $<$ a $4 \mathrm{~mm}$, y este porcentaje sube dramáticamente al $40 \%$ en aquellos que miden más de $9 \mathrm{~mm}$. 


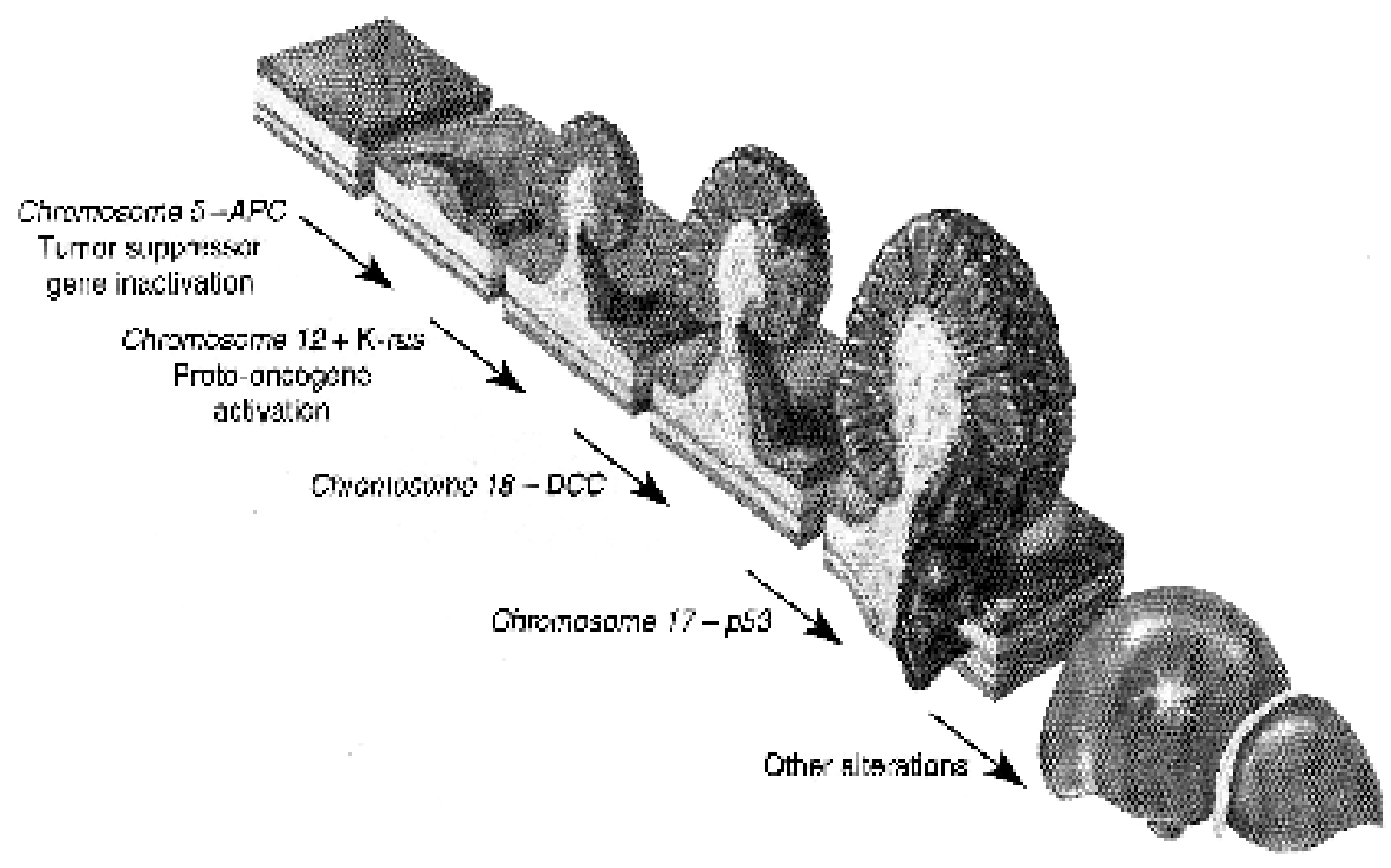

FIGURA 1. Evolución general del adenoma.

\section{Tratamiento}

Se ha demostrado que la aparición de un pólipo demora aproximadamente 5 años; la aparición de un CCR a partir de la malignización de un adenoma previamente existente demora otros 5 años.

La remoción de los pólipos colónicos en forma rutinaria permite una reducción estadísticamente significativa en la incidencia de CCR.

Por lo tanto se considera que la remoción de los pólipos adenomatosos del colon constituye una profilaxis contra el CCR.

Desde la aparición de la colonoscopía, el tratamiento de los pólipos colónicos se ha facilitado mucho, ya que la gran mayoría son resecables con el colonoscopio con una morbimortalidad asociada mínima.

Los pacientes a los que se les ha encontrado pólipos colónicos tienen una alta posibilidad de desarrollar nuevos pólipos por lo que se les recomienda un seguimiento colonoscópico. Una vez que se ha logrado limpiar de pólipos el colon se debe indicar un control colonoscópico dentro de 3 a 5 años.

\section{Adenomas con cáncer invasor}

Se considera cáncer invasor a aquel que compromete más allá de la muscular de la mucosa, y que por lo tanto tiene riesgo de metástasis linfáticas y por lo tanto requiere en la mayoría de los casos de una resección colorectal formal.

En 1985 Haggit propuso una clasificación para pólipos adenomatosos con cáncer invasor, que es la que actualmente está en uso (Figura 2):

Nivel 0: Cáncer in situ o intramucoso (no invasor).

Nivel 1: Cáncer que invade a través de la muscular de la mucosa pero que no sobrepasa la cabeza del pólipo (sobre la unión del pólipo con su pedículo).

Nivel 2: Cáncer que invade el cuello del pólipo (unión entre la cabeza y el pedículo).

Nivel 3: Cáncer que invade cualquier porción del pedículo.

Nivel 4: Cáncer que invade hasta la submucosa de la pared intestinal pero sin infiltrar la muscular propia. Por lo tanto todos los pólipos sésiles con cáncer invasor son nivel 4.

Un pólipo sésil con cáncer invasor tiene un 10\% de posibilidades de invasión linfática. 


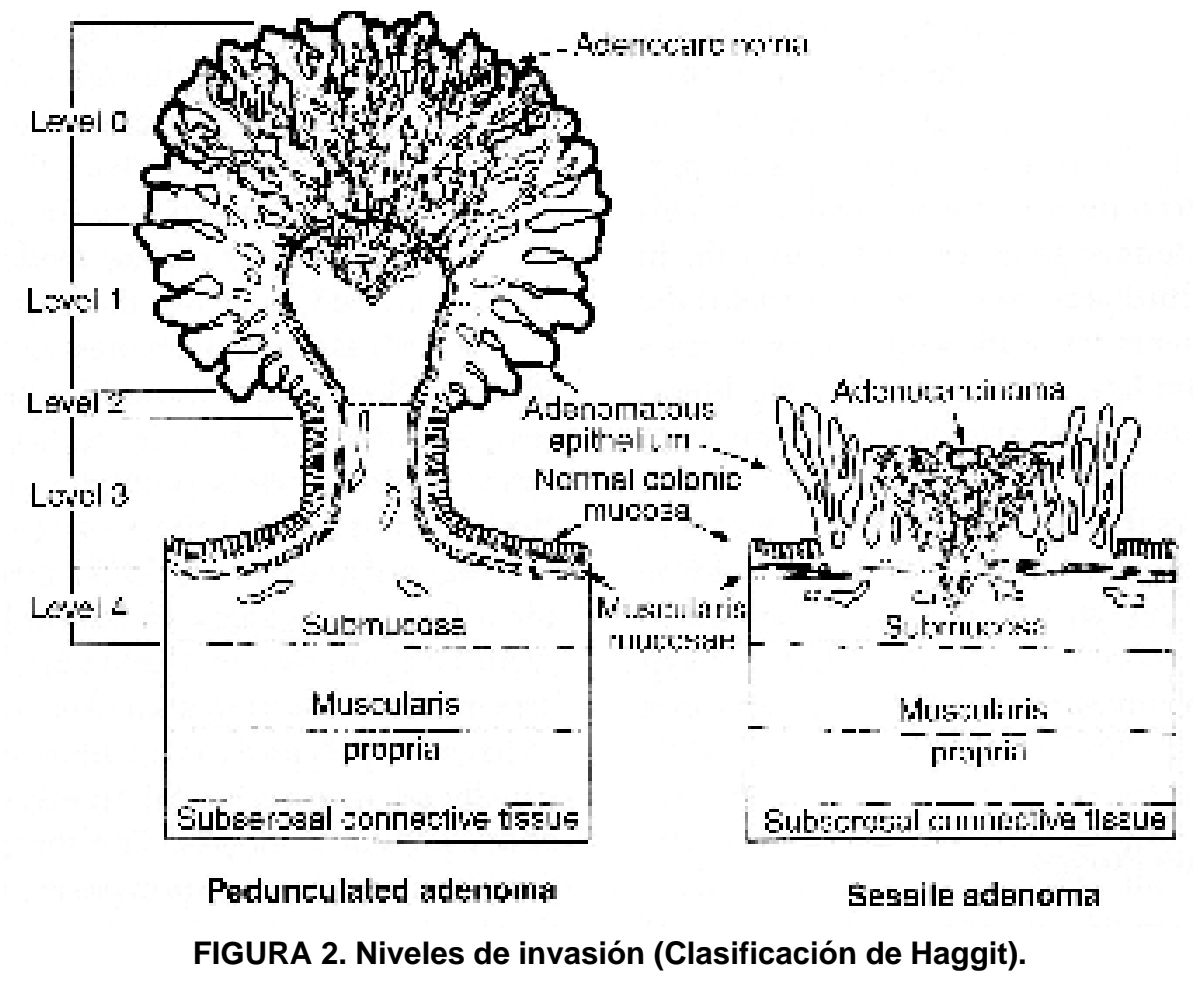

\section{Tratamiento:}

Para aquellos pólipos con cáncer invasor confinado a los niveles $0,1,2$, y 3 basta la polipectomía completa; sin embargo deben ser sometidos a un riguroso programa de seguimiento colonoscópico cada 3 meses durante el primer año, cada 6 meses durante el $2^{\circ}$ y $3^{\circ}$ año y anual hasta completar los 5 años de seguimiento.

Para aquellos que presenten compromiso hasta el nivel 4 (los pólipos sésiles incluidos), y para los que presente factores de riesgo como indiferenciación, compromiso vascular y/o linfático, debieran ser sometidos a una resección quirúrgica formal.

\section{2) Pólipos hamartomatosos:}

Un hamartoma es una malformación o error neonatal caracterizado por la mezcla de tejidos en un sitio que no le corresponde.

\section{a) Pólipos juveniles:}

Ocurren con mayor frecuencia en edad pediátrica, sin exclusión de los adultos. Este tipo de pólipos son hamartomas y por lo tanto no son premalignos. Generalmente son de gran tamaño y por lo tanto su síntoma más frecuente es el sangrado. Son los únicos pólipos que pueden sufrir una autoamputación. Ocasionalmente pueden producir una intosucepción.

\section{b) Poliposis juvenil:}

Es una entidad poco frecuente que se caracteriza por la presencia de cientos de pólipos distribuidos a lo largo de todo el colon, comprometiendo en algunos casos incluso el estómago y/o el intestino delgado. Se describen varias malformaciones asociadas hasta en el $20 \%$ de los casos, como por ejemplo macrocefalia, labio leporino, paladar hendido, porfiria, malformaciones arteriovenosas, malformaciones cardiacas, malrrotación gástrica, etc. Se considera poliposis juvenil a la presencia de tres o más pólipos, y a diferencia de los pólipos juveniles, la poliposis si constituye una entidad con potencial de malignización.

Debido a lo anterior el enfrentamiento quirúrgico es el mismo de la poliposis adenomatosa familiar, es decir se realiza una colectomía subtotal con ileo- recto anastomosis o de preferencia una proctocolectomía con reservorio ileal en

"J". Los familiares de primer grado deben ser seguidos colonoscopicamente desde la $2^{\circ}$ década aún si son asintomáticos. 


\section{c) Síndrome de Peutz-Jeghers:}

Constituye una entidad hereditaria caracterizada por la presencia de pólipos hamartomatosos en el tubo digestivo, asociado a la presencia de manchas melanocíticas en la mucosa bucal y labial. El compromiso facial y en extremidades de estas manchas es variable, pero el compromiso bucal es una condición sinequa non.

El compromiso del intestino delgado es una constante en este síndrome. Sin embargo el compromiso del estómago, colon y recto son variables.

Desde el punto de vista histológico esto pólipos presentan una distribución de la muscular de la mucosa dentro de la lámina propia de tal manera que asemeja un árbol de navidad.

Los síntomas más frecuentes son el dolor abdominal, el sangrado digestivo, y la obstrucción intestinal.

Si bien los pólipos hamartomatosos no se consideran premaliagnos, está ampliamente demostrado que los pacientes portadores del síndrome de Peutz-Jeghers tienen mayor riesgo de presentar cánceres tanto del la esfera digestiva, como de la extra digestiva.

Dentro de los cánceres digestivos el más frecuente es el pancreático. Dentro de los extra digestivos, los más frecuentes son mama, ovario cuello y testicular.

EI CCR en los pólipos de Peutz-Jegherz se puede presentar ya sea por malignización hamartomatosa, o por la malignización de pólipos adenomatosos concomitantes.

\section{Tratamiento:}

Todos los pólipos > a $5 \mathrm{~mm}$ deben ser removidos endoscópicamente (tanto gástricos como colo-rectales). Todo pólipo mayor de 15 $\mathrm{mm}$ en el intestino delgado debe ser removido quirúrgicamente.

\section{Seguimiento:}

Estos pacientes deben ser controlados anualmente con hemograma, examen ginecológico y mamario, papanicolao, ecografía pélvica, testicular y abdominal. Endoscopía alta y baja deben ser realizadas cada dos años.

\section{d) Síndrome de Cronkhite-Canada:}

Se caracteriza por una poliposis difusa del tubo digestivo asociado a alopecia, pigmentaciones cutáneas y atrofia de las uñas de los pies y manos. Se presenta en las década de los 70, no es hereditario y los pólipos son hamartomatosos.

Sus síntomas principales son diarrea intensa con enteropatía perdedora de proteínas, vómitos, baja de peso y desnutrición.

Desde el punto de vista histológico, los pólipos contienen dilataciones quísticas similares a los de los pólipos juveniles, pero los pólipos son más pequeños y carecen del exceso de lámina propia.

El desarrollo de cáncer en este tipo de pólipos, si bien ha sido descrito, es raro.

La resección intestinal esta reservada solo para casos con complicaciones como la obstrucción intestinal.

\section{e) Enfermedad de Cowden:}

Consiste en un raro síndrome familiar con hamartomas endo, ecto y mesodérmicos.

Los pólipos en este síndrome son pequeños $(<5 \mathrm{~mm})$. El factor de mayor importancia en relación a estos pólipos consiste en su asociación con otras lesiones, particularmente con carcinoma mamario, tiroideo, quistes ováricos, y otros pólipos gastrointestinales. Debido a que su riesgo de malignización es nulo, no es necesario su remoción. Sólo se recomienda el control colonoscópico cada 3 a 5 años.

\section{3) Pólipos inflamatorios:}

a) Pseudopólipos: Si bien microscópicamente son muy semejantes a lo pólipos adenomatosos, al examen microscópico se observan islotes de mucosa normal junto con mucosa con proceso inflamatorio.

Son producidos por procesos inflamatorios previos tales como colitis ulcerosa, enfermedad de Crohn, colitis amebiana, o isquémica, lo que puede resultar en zonas de pérdida parcial e la mucosa concomitantes con otras áreas de mucosa conservada, las que adquieren el aspecto de un pólipo. Muchas veces es difícil establecer la diferencia macroscópica entre un pesudopólipo y un pólipo adenomatoso, o incluso de una poliposis adenomatosa familiar, por lo que la biopsia es fundamental. Estos pesudopólipos carecen de potencial maligno.

b) Pólipos linfoídeos: Constituyen crecimientos de folículos linfáticos, y se presentan con mayor frecuencia en el recto. Pueden ser solitarios o múltiple, por lo que no deben confundirse co ua poliposis adenmatosa familiar. 
Su causa es aún desconocida. Los criterios histológicos para establecer el diagnóstico fueron establecidos por Dawson y Morson, según los cuales, el tejido linfático debe estar completamente dentro de la mucosa y submucosa y sin existir invasión a la capa muscular. Si la muestra no incluye la muscular se considera que el diagnóstico no puede ser concluyente.

\section{4) Pólipos hiperplásicos:}

Los pólipos hiperplásicos también son denominados metaplásicos. No tienen potencial de malignización. Se encuentran con frecuencia en la mucosa del colon como pequeños nódulos de la mucosa de entre 3 a $5 \mathrm{~mm}$ de diámetro (a pesar que ocasionalmente se les puede encontrar de mayor tamaño en las porciones más proximales del colon). La gran mayoría se encuentra ya sea en el recto o en el sigmoides.
Existen varios reportes de pólipos mixtos (hiperplásicos y adenomatosos), los que debieran ser considerados como pólipos neoplásicos, considerando su potencial de malignización, y por lo tanto resecados y sometidos al mismo esquema de seguimiento que los pólipos adenomatosos. La posibilidad de considerar a los pólipos hiperplásicos como marcadores para la presencia de adenomas en el colon ha sido muy cuestionada ya que su valor predictivo es muy bajo.

\section{CONCLUSIÓN}

Los pólipos en el colon son muy frecuentes, pero de tipos y comportamientos muy diversos. Un conocimiento adecuado de ellos permite otorgarles la importancia que les corresponde e incluso contribuir en la profilaxis del CCR.

\section{BIBLIOGRAFÍA}

1) Tjandra JJ, Kilkenny JW, Buie WD, Hyman N, Simmang C, Anthony T, et al. The Standards Practice Task Force; The American Society of Colon and Rectal Surgeons. Practice Parameters for the Management of Rectal Cancer. Dis Col Rectum 2005; 48:411-23.

2) Jemal A, Tiwari RC, Murray T, Ghafoor A, Samuels A, Ward E, et al. American Cancer Society. Cancer statistics 2004. CA Cancer J Clin 2004; 54:8-29.

3) Donoso A, Villarroel L, Pinedo G. Aumento de la mortalidad por cáncer de colon en Chile, 19902003. Rev med Chil 2006;134: 152-4.

4) Dukes $C$. Simple tumors of the large intestine and their relationship to cancer. Br J Surg 1926; 13:720-33

5) Jackman RJ, Mayo CW. The adenomacarcinoma secuence in cancer of the colon. Surg Gynecol Obstet 1951; 93:327-30

6) Gordon, P, Nivatvongs, S. Principles and practice of surgery of the colon, rectum and anus. Quality Medical Publishing, Inc. St Louis, Missouri. 1999.

7) Feig, B, Berger, D, Fuhrmann, G. The M.D. Anderson surgical oncology handbook.

8) Stryker SJ, Wolf WG, Culp CE, Libbe SD, Ilstrup DM, MAC-Carty RL. Natural history of untreated colonic polyps. Gastroenterology 1987; 93:1009-13. 